\title{
Synaptic Control of Spiking in Cerebellar Purkinje Cells: Dynamic Current Clamp Based on Model Conductances
}

\author{
Dieter Jaeger ${ }^{1}$ and James M. Bower ${ }^{2}$ \\ 1Department of Biology, Emory University, Atlanta, Georgia 30322, and 2Division of Biology, California Institute of \\ Technology, Pasadena, California 91125
}

Previous simulations using a realistic model of a cerebellar Purkinje cell suggested that synaptic control of somatic spiking in this cell type is mediated by voltage-gated intrinsic conductances and that inhibitory rather than excitatory synaptic inputs are more influential in controlling spike timing. In this paper, we have tested these predictions physiologically using dynamic current clamping to apply model-derived synaptic conductances to Purkinje cells in vitro. As predicted by the model, this input transformed the in vitro pattern of spiking into a different spike pattern typically observed in vivo. A net inhibitory synaptic current was required to achieve such spiking, indicating the presence of strong intrinsic depolarizing currents. Spiketriggered averaging confirmed that the length of individual intervals between spikes was correlated to the amplitude of the inhibitory conductance but was not influenced by excitatory inputs. Through repeated presentation of identical stimuli, we determined that the output spike rate was very sensitive to the relative balance of excitation and inhibition in the input conductances. In contrast, the accuracy of spike timing was dependent on input amplitude and was independent of spike rate. Thus, information could be encoded in Purkinje cell spiking in a precise spike time code and a rate code at the same time. We conclude that Purkinje cell responses to synaptic input are strongly dependent on active somatic and dendritic properties and that theories of cerebellar function likely need to incorporate single-cell dynamics to a greater degree than is customary.

Key words: cerebellum; Purkinje cell; synapse; excitation; inhibition; dynamic clamp; modeling; in vitro; whole cell
The near-crystalline anatomical organization of cerebellar cortex has traditionally been the main impetus for theoretical considerations of cerebellar function (Marr, 1969; Albus, 1971). In these theories, single neurons are primarily treated as integrate-andfire devices, a treatment that is still customary in large-scale theories of network function. Over the last 20 years, however, it has been well established that Purkinje cells have complex intrinsic dynamical properties (Llinás and Sugimori, 1980a, 1992). Most notably, a large voltage-gated calcium conductance is capable of producing dendritic plateau potentials and calcium spikes (Llinás and Sugimori, 1980b). The Purkinje cell takes a central position in theories of cerebellar function, because it integrates the various sources of input to cerebellar cortex and provides its only source of output. Therefore, a large contribution of active intrinsic properties of Purkinje cells in synaptic integration could be a key element in the elusive algorithm of cerebellar cortical computation. To examine how spike responses may be under the combined control of intrinsic properties and synaptic inputs, we use an interactive approach of computer modeling and physiological recordings.

In previous studies, we have developed a realistic model of a single Purkinje cell to establish a basis for considering the functional significance of its active properties (De Schutter and Bower, 1994a). The model was constructed using the morphology of a real Purkinje cell (Rapp et al., 1994) and includes the full set

Received Jan. 29, 1999; revised April 22, 1999; accepted April 26, 1999.

This work was supported by a Sloan fellowship to D.J., by a grant from the Human Frontier Science Program to J.M.B., and by National Institute of Mental Health Grant MH57256-01 to D.J. We thank Erik De Schutter for helpful comments and simulation scripts.

Correspondence should be addressed to Dr. Dieter Jaeger, Department of Biology, 1510 Clifton Road, Emory University, Atlanta, GA 30322.

Copyright (C) 1999 Society for Neuroscience 0270-6474/99/196090-12\$05.00/0 of voltage-gated conductances identified to date in this neuron. It was tuned to replicate physiological current injection data obtained from the brain-slice preparation (De Schutter and Bower, 1994a) and was subsequently tested by comparing its response to synaptic input with the activity found in real in vivo Purkinje cells (De Schutter and Bower, 1994b). It was found that the model quite closely replicates typical Purkinje cell responses to synaptic input (De Schutter and Bower, 1994c).

Recently, we have more closely analyzed the way in which synaptic input influences the somatic spiking of the model (Jaeger et al., 1997). We found that the model could only generate typical in vivo spiking patterns, when the average inhibitory synaptic current exceeded the excitatory current. This requires the presence of a continuous baseline of inhibitory synaptic input. Furthermore, the model predicts that the largest contribution to fluctuations in the membrane potential in the soma as well as the dendritic tree is made by the large intrinsic voltage-dependent currents rather than by the currents associated with either excitatory or inhibitory synaptic inputs.

Modeling predictions rest on many assumptions made in the construction of the model (Bhalla and Bower, 1993). Therefore, modeling results need to be tested experimentally before they can be accepted. The present study was designed to allow such testing of our model predictions regarding the involvement of active cell properties in synaptic integration. To replicate the conditions of the model as closely as possible, we chose to apply synaptic conductances generated by the model to Purkinje cells in vitro using the dynamic current-clamp technique (Sharp et al., 1993). As in the model, the addition of a stochastic baseline of excitatory and inhibitory input resulted in a transition from an in vitro bursting spike pattern to ongoing irregular spiking, which closely resembles spontaneous spiking recorded in vivo (Jaeger and 
Bower, 1994). The condition of continuous random input used here and in our previous modeling study (Jaeger et al., 1997) reflects our best estimate of synaptic activity expected to be present as a continuous baseline in vivo. Because little is known about the specific input modulations occurring during taskrelated behavior, we restrict our analysis to a stochastic activation of all inputs. By manipulating the balance and amplitude of excitatory and inhibitory inputs, we address the question of how output spike rate and spike timing reflect synaptic input patterns in the presence of large intrinsic active properties.

\section{MATERIALS AND METHODS}

Slice preparation and whole-cell recording. All animal procedures fully complied with the National Institutes of Health guidelines on animal care and use. Whole-cell recordings were obtained from the somata of Purkinje cells in $300 \mu \mathrm{m}$ sagittal slices from 2- to 4-week-old rats under visual control using a fixed-stage microscope. The slice medium contained (in mM): $\mathrm{NaCl} 124, \mathrm{KCl} 5, \mathrm{NaHCO}_{3} 26, \mathrm{~K}_{2} \mathrm{HPO}_{4} 1.2, \mathrm{CaCl}_{2} 2.4$, $\mathrm{MgSO}_{4}$ 1.3, and glucose 10. Electrodes were filled with (in mM): K gluconate 120, $\mathrm{KCl}$ 10, HEPES 10, EGTA 10, CaCl 2, MgCl 2, Na-ATP 2, and Na-GTP 0.2, with impedances ranging from 6 to $12 \mathrm{M} \Omega$. Recordings were obtained at $32^{\circ} \mathrm{C}$. To study the effects of simulated synaptic input in isolation, we blocked endogenous excitatory and inhibitory inputs with $5 \mu \mathrm{M}$ CNQX and $40 \mu \mathrm{M}$ picrotoxin, respectively.

Implementation of the dynamic clamp. Synaptic conductances obtained from our Purkinje cell model were applied to Purkinje cells in vitro using the dynamic current-clamp method (Robinson and Kawai, 1993; Sharp et al., 1993). We implemented this technique using dual whole-cell recordings in which one electrode was used to record membrane potential $\left(\mathrm{V}_{\mathrm{m}}\right)$ and the other was used to inject simulated synaptic current. The recording electrode was connected to channel 1 on an Axoclamp 2A amplifier, and the feedback current injection was applied through channel 2 of this instrument. The two-electrode approach was chosen because it is not possible to inject sizeable fluctuating currents through an electrode while maintaining an accurate voltage recording with the same electrode. In the feedback condition of dynamic clamping, this problem can easily lead to ringing artifacts. The amount of simulated synaptic current injected $\left(I_{\mathrm{inj}}\right)$ was calculated at a $10 \mathrm{kHz}$ refresh rate from the recorded membrane potential $\left(\mathrm{V}_{\mathrm{m}}\right)$ and two stored synaptic conductance waveforms representing the sum of excitatory $\left(G_{\mathrm{ex}}\right)$ and inhibitory $\left(G_{\mathrm{in}}\right)$ conductances obtained from computer simulations. The equation to calculate synaptic current is: $I_{\mathrm{inj}}=I_{\mathrm{ex}}+I_{\mathrm{in}}=G_{\mathrm{ex}} *\left(E_{\mathrm{ex}}-\mathrm{V}_{\mathrm{m}}\right)+G_{\mathrm{in}} *\left(E_{\mathrm{in}}-\right.$ $\left.\mathrm{V}_{\mathrm{m}}\right)$, where $E_{\mathrm{ex}}$ and $E_{\mathrm{in}}$ are the synaptic reversal potentials of $0 \mathrm{mV}$ for excitation and $-70 \mathrm{mV}$ for inhibition, respectively. The recorded membrane potential $\left(\mathrm{V}_{\mathrm{m}}\right)$ was low-pass filtered at $5 \mathrm{kHz}$ using the Axoclamp $2 \mathrm{~A}$ output filter before the potential was fed to the dynamic-clamp software to prevent possible aliasing. This software was custom written in Turbo Pascal with in-line assembly code for fast feedback control under the DOS operating system. It works in conjunction with a CIO-DAS $16 / \mathrm{F}$ analog-to-digital (AD) board (Computer Boards), which uses an onboard clock to time simultaneous AD conversion and digital-to-analog (DA) output. The acquisition program polls the $\mathrm{AD}$ conversion and calculates the feedback equation for the next time step while all external interrupts are disabled. The system was tested to be reliable for $20 \mathrm{kHz}$ feedback rates with artificial input voltages consisting of square voltage pulses. The DAS 16/F DA converters put out voltages from 0 to $5 \mathrm{~V}$. A hardware device was constructed to subtract $2.5 \mathrm{~V}$ from this signal and to provide low-pass filtering at a cutoff frequency of $10 \mathrm{kHz}$. This filtering was needed to eliminate very-high-frequency noise generated by the computer. The resulting output voltage from -2.5 to $2.5 \mathrm{~V}$ was used as input to the "current command" BNC connector on the back of the Axoclamp 2A amplifier. Because an HS2A 0.1LU head stage was used, the command voltage allowed dynamic current clamping for maximal current values of $\pm 2.5 \mathrm{nA}$. The maximal value of $+2.5 \mathrm{nA}$ was never reached, whereas $-2.5 \mathrm{nA}$ was injected transiently at the peak of somatic spikes in some recordings.

Recording stability and potential experimental problems. Data acquisition was started directly after a dual whole-cell configuration was obtained and was continued for as long as the physiological characteristics of the cell remained stable. The characteristics used were spike size $(>45$ $\mathrm{mV}$ ), a stable spike rate during dynamic-clamp stimuli, and the absence of depolarization-induced spike inactivation. Although a gradual change in cell properties might be expected because of the perfusion with the pipette solution, such a change was not observed for the spike responses with dynamic clamping examined here. On theoretical grounds, a junction potential of $\sim 10 \mathrm{mV}$ can be expected for recording solutions based on K gluconate (Barry, 1994). This junction potential, however, can be mostly offset by a Donnan potential of opposite sign (Barry and Lynch, 1991). The large intracellular anions responsible for this Donnan potential equilibrate with the electrode solution over a few minutes in small cells (Pusch and Neher, 1988). This equilibration may not occur to a significant degree in large cells, such as Purkinje cells, during the period of time over which we acquired data. We examined a possible voltage offset in our whole-cell recordings by comparing the observed spike threshold with that of previous recordings we obtained in the same preparation with sharp electrodes (Jaeger and Bower, 1994). We found no consistent offset in spike threshold between the two methods of recording. Therefore we did not subtract a junction potential from the recorded $\mathrm{V}_{\mathrm{m}}$ before feeding it into the dynamic clamp. We cannot exclude some error in the absolute membrane potential recorded in individual cells. With respect to dynamic current clamping, an offset in recorded $\mathrm{V}_{\mathrm{m}}$ is equivalent to shifting the synaptic reversal potentials of excitation and inhibition by the same offset. Therefore differences in the absolute level of depolarization and the spike rate of different neurons obtained with the same input pattern should be interpreted with care.

The use of $10 \mathrm{~mm}$ EGTA in our electrode solution could potentially reduce the activation of $\mathrm{Ca}$-dependent $\mathrm{K}$ conductances in the cell by buffering the required $\mathrm{Ca}$. Thus intrinsic hyperpolarizing currents could be diminished. In a later set of experiments (D. Jaeger, unpublished observations), the internal solution was changed to contain only $0.1 \mathrm{~mm}$ EGTA. The results of these later experiments show that the intrinsic balance of inward and outward currents during dynamic current clamping is in the same range with 0.1 or $10 \mathrm{~mm}$ EGTA in the recording pipette. The lack of an effect of EGTA can be explained by its slowbuffering characteristics and by the possible activation of Ca-dependent $\mathrm{K}$ channels via specific $\mathrm{Ca}$ channels located in close proximity. Unfortunately, the dynamics of calcium handling in Purkinje cells is yet poorly understood.

The construction of simulated synaptic conductances. The synaptic conductance patterns that we applied in the present study via dynamic clamping were computed with our Purkinje cell model and were identical to the inputs we used in our previous modeling study (Jaeger et al., 1997). The model was constructed to replicate the best estimate in the number and location of excitatory parallel fiber and inhibitory stellate cell inputs to a single Purkinje cell. The modeling methods used to generate synaptic conductances were described in detail in our previous publications (De Schutter and Bower, 1994a,b; Jaeger et al., 1997). In brief, the excitatory input from a granule cell was modeled as an alpha function with an opening time constant of $0.5 \mathrm{msec}$, a closing time constant of 1.2 msec, a reversal potential of $0 \mathrm{mV}$, and a fixed maximal conductance of $0.7 \mathrm{nS}$. Inhibitory inputs as expected from stellate cell input had an opening time constant of $0.9 \mathrm{msec}$, a closing time constant of $9 \mathrm{msec}$, a reversal potential of $-70 \mathrm{mV}$, and a variable amplitude proportional to the surface area of the postsynaptic compartment (resulting in a mean amplitude of $3 \mathrm{nS}$ ). The reversal potential of inhibitory input was shifted by $10 \mathrm{mV}$ from the value of $-80 \mathrm{mV}$ used previously in the model, which is the only difference between synaptic conductance patterns used here and those in our modeling study (Jaeger et al., 1997). Each synapse was activated randomly at a specified mean rate, which resulted in an exponential distribution in the duration of intervals between inputs. All excitatory synapses were activated with the same mean rate, as were all inhibitory synapses. The rate of activation of each of the 1474 excitatory synapses was increased compared with the expected in vivo situation to compensate for the reduced number of synapses present in the model (De Schutter and Bower, 1994b; Jaeger et al., 1997). To apply synaptic conductances through a single electrode with dynamic current clamping, we pooled conductances from all synapses in the model by summing. On average, conductance waveforms consisted of 848 inhibitory and 16,225 excitatory inputs per second (Jaeger et al., 1997).

The choice of synaptic parameters. In our original modeling efforts, the number of parallel fiber inputs to a single Purkinje cell were based on known anatomical data (Harvey and Napper, 1988). The location of synapses on the dendrite was based on EM data (Palay and Chan-Palay, 1974). The number of stellate cell inputs and the choice of conductance parameters for single inputs were partly based on best-guess estimates, however (De Schutter and Bower, 1994b). Since the original model was constructed, several groups have reported amplitudes and time constants for both excitatory (Barbour, 1993) and inhibitory (Vincent et al., 1992; 
Barbour, 1993; Häusser and Clark, 1997) synaptic inputs to Purkinje cells. These data show that the original values chosen in the model were quite accurate, except for the decay time constant of inhibitory inputs. In the modeling and experimental results reported in this paper, this decay time constant was shortened from 26 to $9 \mathrm{msec}$ to conform with the experimental finding (Vincent et al., 1992). The number of 1695 inhibitory synapses used in the model was approximately realistic (Jaeger et al., 1997; Sultan and Bower, 1998).

The combined reversal potential of excitatory and inhibitory conductances defines a partial voltage clamp. The combined reversal potential of excitatory and inhibitory inputs $\left(E_{\text {tot }}\right)$ applied with dynamic clamping can be calculated as the weighted sum of the reversal potentials of excitation and inhibition: $E_{\mathrm{tot}}=\left(G_{\mathrm{ex}} * E_{\mathrm{ex}}+G_{\mathrm{in}} * E_{\mathrm{in}}\right) /\left(G_{\mathrm{ex}}+G_{\mathrm{in}}\right)$. This combined reversal potential $\left(E_{\text {tot }}\right)$ was termed $\mathrm{V}_{\text {clamp }}$ in our modeling publication (Jaeger et al., 1997) because the dynamic clamp creates a voltage clamp such that any deviation in membrane potential from $\mathrm{V}_{\text {clamp }}$ is counteracted by an opposing change in synaptic current. If $\mathrm{V}_{\mathrm{m}}$ followed this trajectory of $V_{\text {clamp }}$ exactly, no synaptic current would be injected at any time. As with a voltage-clamp amplifier, the amplitude of injected current is proportional to the deviation of $\mathrm{V}_{\mathrm{m}}$ from $\mathrm{V}_{\text {clamp }}$. The gain of the voltage clamp exerted by synaptic input corresponds to the total amplitude of synaptic conductance $G_{\mathrm{ex}}+G_{\mathrm{in}}$. Because this gain is rather low compared with a regular voltage clamp and easily allows the escape of $V_{m}$ from $V_{\text {clamp }}$, we refer to dynamic clamping as a partial voltage clamp. This analysis also applies to a baseline of continuous synaptic input conductances expected to be present in vivo.

The validity of using focalized input to examine the function of natural inputs. The technique of dynamic current clamping requires that simulated synaptic input be applied via one command current at a single point in the cell. In contrast, Purkinje cells in vivo receive distributed synaptic input. Distributed input is the situation we recreated in our previous modeling study (Jaeger et al., 1997) to examine the interaction of intrinsic cell properties with synaptic input under conditions expected in vivo. To interpret the results of dynamic current clamping, it is crucial to establish the effects of focalizing synaptic input at the soma in comparison with distributed dendritic inputs. In the Purkinje cell model, the effect of distributed dendritic input is critically dependent on the activation of dendritic voltage-gated currents (Jaeger et al., 1997). Therefore we need to establish whether synaptic input focalized at the soma can still evoke these dendritic currents.

When only the passive properties of Purkinje cells are considered, these cells are electrotonically very compact. The average length from the soma to the tip of a dendrite is only 0.13 length constants (Rapp et al., 1994). Therefore, a prolonged current injected into the soma will lead to a nearly uniform change in membrane potential throughout the cell (Rall, 1959). The dendritic attenuation increases for higher frequencies of changes in membrane potential $\left(\mathrm{V}_{\mathrm{m}}\right)$ at the soma, however (Spruston et al., 1993). As a result, dendritic $\mathrm{V}_{\mathrm{m}}$ follows slow fluctuations $(<10 \mathrm{~Hz})$ in somatic $V_{m}$ very well, whereas fast fluctuations $(>100 \mathrm{~Hz})$ are severely attenuated in the distal dendrites (Spruston et al., 1993). Even for a total dendritic length of 1.0 length constants, this relationship still holds. This is the maximum length expected for Purkinje cell dendrites in the presence of voltage-gated conductances (De Schutter and Bower, 1994a). The fluctuations in our simulated synaptic input are almost entirely contained in the frequency band from 0 to $100 \mathrm{~Hz}$. Thus, a large part of this signal is expected to spread to most of the dendritic tree and to influence the activation of dendritic conductances. To establish in detail how much the difference between distributed and focalized input may affect Purkinje cell responses, we analyzed the results from both input conditions in the model.

Figure 1 compares the model's response when synaptic input was present throughout the dendrite $(A-C)$ with data obtained when synaptic input was focalized at the soma as is the case with dynamic current clamping $(D-F)$. The focalized input was obtained by summing all synaptic conductances of the simulation using distributed synapses and by applying the sum to the soma. To achieve a spike rate with focalized input that matched the spike rate observed with distributed input, we adjusted the reversal potential of inhibitory input from -80 to $-72 \mathrm{mV}$. This requirement for a less-hyperpolarized inhibitory reversal potential indicates that inhibitory input focalized at the soma is somewhat more effective in suppressing spiking than is distributed dendritic input. Note that the inhibitory reversal potential of $-72 \mathrm{mV}$ in the focalized model is quite close to the $-70 \mathrm{mV}$ we used during dynamic current clamping. Shifting the reversal potential of inhibition between the distributed and focalized input conditions allowed us to use the same conductance patterns while retaining a matching spike rate. The resulting voltage traces show that both types of input induced the irregular pattern of spiking characteristic of the in vivo state (see Figs. $1 A$, 3). Furthermore, the total synaptic current and the evoked intrinsic voltage-gated currents (Fig. $1 C, F$ ) were very similar in both cases. This means that the focalized synaptic inputs at the soma still resulted in the activation of dendritic voltage-gated currents. In both cases the voltage-gated conductances in the dendrite produced a sustained inward current $\left(I_{\mathrm{Ca}+\mathrm{K}}\right.$; Fig. $\left.1 C, F\right)$. This current resulted in a similar plateau depolarization of the dendrite for distributed and focalized input (Fig. 1 $A, D$ ). The mean outward synaptic current (Fig. 1C,F) indicates that the depolarizing currents in the cell pushed $\mathrm{V}_{\mathrm{m}}$ in the soma above the net synaptic reversal potential. Therefore, the net function of synaptic conductances was to counteract intrinsic inward conductances. Although the exact timing of individual spikes was different for the distributed and focalized synaptic input, periods of increased spike rate clearly matched. In both input conditions, these periods of spiking were carried by a relative increase of inward dendritic current (horizontal bars in Fig. $1 C, F$ ). These similarities indicate that dendritic input in Purkinje cells does not lead to large nonlinear local effects in the dendrite. An absence of differences in cell response to clustered or distributed dendritic input was observed previously in the model (De Schutter and Bower, 1994c). As may be expected from the preferential dendritic attenuation of high-frequency components in somatic input, focalized input resulted in maintained slow fluctuations in dendritic responses compared with that in distributed input. In contrast, the fastest components of the response to input were partly different between the two conditions.

The observed similarities in the response of the model to focalized or distributed synaptic input suggest that dynamic current clamping is a reasonable approximation to distributed synaptic input for the modeling predictions we want to test. We therefore proceed to use this method to apply model-based synaptic input patterns to Purkinje cells in vitro.

\section{RESULTS Stochastic synaptic inputs are sufficient to elicit in
vivo spike patterns in slice recordings}

As shown in Figure $2 A$ and in previous publications (Llinás and Sugimori, 1980a,b), in vitro cerebellar Purkinje cells exhibit spontaneous periods of fast regular somatic spiking that gradually lead to dendritic calcium spike bursting. In contrast, in vivo patterns of spiking are slower, more irregular, and never include spontaneous calcium spikes (Jaeger and Bower, 1994).

Our modeling results predict that the addition of continuous excitatory and inhibitory synaptic input converts the spontaneous in vitro spike pattern of Purkinje cells to an in vivo spike pattern (De Schutter and Bower, 1994b; Jaeger et al., 1997). This modeling prediction was tested here by applying the same conductances used in the model via the dynamic clamp in vitro. We found that the in vitro spike pattern (Fig. $2 A$ ) was immediately transformed into irregular ongoing spiking (Fig. $2 C$ ). In contrast, when only excitatory inputs were applied with the dynamic clamp (Fig. $2 B$ ), the calcium spike burst pattern of in vitro neurons was enhanced in all cells tested $(n=8)$. Similar results were obtained from the model (Jaeger et al., 1997).

A comparison of interspike interval (ISI) histograms documents the similarity between in vivo spiking patterns (Fig. $3 A$ ) and those obtained in the model (Fig. $3 B, C$ ) and in the in vitro dynamic current-clamp experiments (Fig. $3 D$ ). In each case, ISI distributions showed a sharp modal peak followed by a tail of longer intervals. Nevertheless, there is not a perfect match between ISI histograms from the model and the data collected in vivo or in vitro. In particular, the exponential falloff from the modal peak appears to be somewhat less steep in the recordings than in the simulations. This may indicate that the activation of voltage-gated currents that underlies periods of fast spiking in the model (Jaeger and Bower, 1994) does not follow exactly the 


\section{Distributed synaptic input}

A

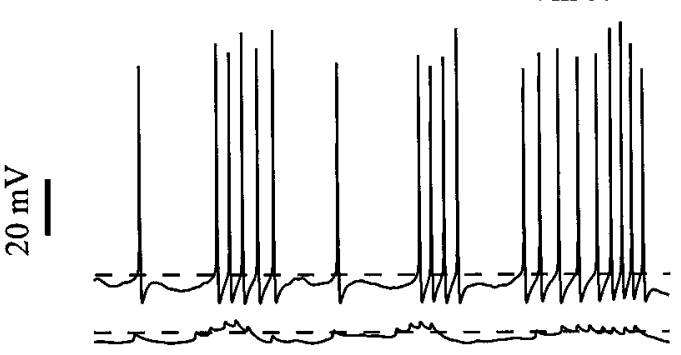

Focalized synaptic input

D

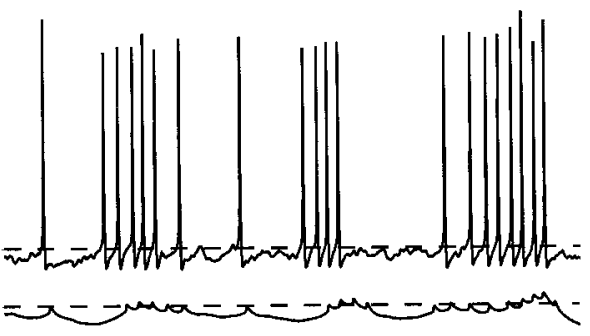

B

Vm dend

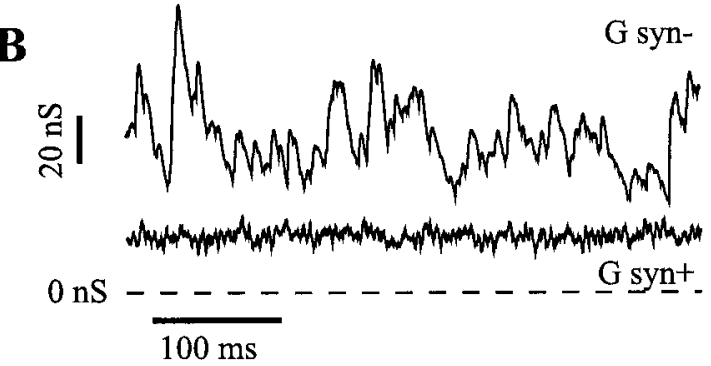

E

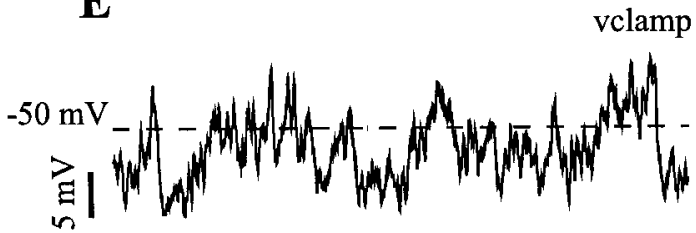

C outward
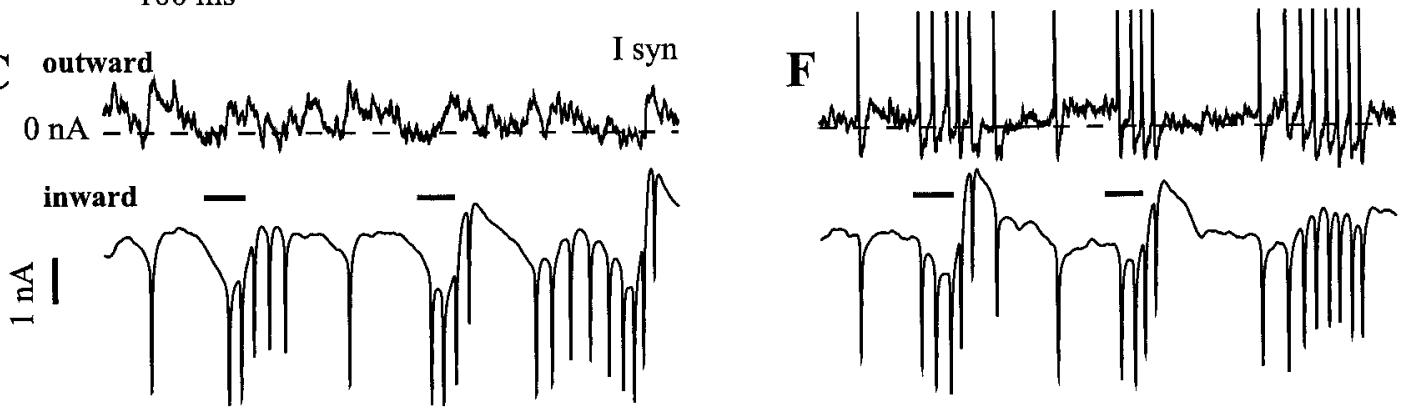

$\mathrm{I} \mathrm{Ca}+\mathrm{K}$

Figure 1. Comparison between distributed $(A-C)$ and focalized $(D-F)$ synaptic input applied to the model. $A, D$, Top, Somatic membrane potential. Bottom, Dendritic membrane potential averaged over all dendritic compartments. Somatic spikes have only a small effect on the averaged dendritic $\mathrm{V}_{\mathrm{m}}$ because of the pronounced dendritic attenuation of high-frequency signals. It should be noted that the main frequency component of an action potential is $\sim 1000 \mathrm{~Hz}$, which is much higher than the main frequencies of synaptic input. dend, Dendrite. $B$, Total excitatory $\left(G_{\text {syn }+}\right)$ and inhibitory $\left(G_{\text {syn }-}\right)$ synaptic conductance obtained by adding up conductances from all synapses in the distributed model. $E$, Combined reversal potential for the synaptic input $\left(V_{\text {clamp }}\right)$ in the focalized case. If the somatic $\mathrm{V}_{\mathrm{m}}$ matched the trajectory of this potential, no synaptic current would be injected. Note that spike responses in the model coincide with depolarized phases in vclamp (horizontal bars in $C, F)$. $C$, $F$, Total synaptic current $\left(I_{\text {syn }}\right)$ and dendritic voltage-gated channel current $\left(I_{\mathrm{Ca}+\mathrm{K}}\right)$ seen in the simulations. The spikes of synaptic current seen during action potentials in the focal case $(F)$ were caused by the large change in synaptic-driving force during somatic action potentials. Nevertheless, the synaptic spike current was much smaller than the somatic Na and $\mathrm{K}$ spike currents and had little effect on the results of the simulations.

identical kinetics as in the cells for which recordings are shown. Note that the identical synaptic input used in the model produced an ISI distribution in vitro that more closely resembles the in vivo recording than the modeling results.

\section{In vivo-type spiking behavior requires a net outward synaptic current}

Our modeling results further predicted that in vivo spiking patterns are specifically dependent on synaptic inputs summing to a mean outward (inhibitory) current (Jaeger et al., 1997). As shown in Figure 4, this prediction is supported by the dynamic-clamp experiments. In all recordings $(n=20)$, a mean outward synaptic current was necessary to generate in vivo-type spike patterns. The net outward synaptic current trace shown in Figure $4 D$ is very similar to that seen in simulations (Fig. 1). For nine cells analyzed quantitatively with the same stimulus, the mean outward current during in vivo-type spiking was $-0.45 \mathrm{nA}$ (SD between cells, 0.08 $\mathrm{nA})$. This hyperpolarizing synaptic current indicates that on average intrinsic depolarizing currents push the cell's membrane potential above the combined reversal potential of inhibition and excitation (Fig. $4 C$ ). In fact, the recorded $\mathrm{V}_{\mathrm{m}}$ was on average 1.3 $\mathrm{mV}$ more depolarized than was the combined synaptic reversal potential (Fig. $4 B, C$ ). This result indicates that in the real cell, as in the model, intrinsic inward plateau currents produce the depolarization required to reach spike threshold and that inhibitory synaptic input acts to limit the activation of these conductances. Candidates for the required noninactivating inward conductances were identified as a dendritic P-type calcium conductance and a somatic persistent $\mathrm{Na}$ conductance by Llinás and Sugimori (1980b, 1992). In the absence of inhibitory input, the activation of these conductances is unstable because of positive feedback, which ultimately leads to dendritic calcium spiking. 

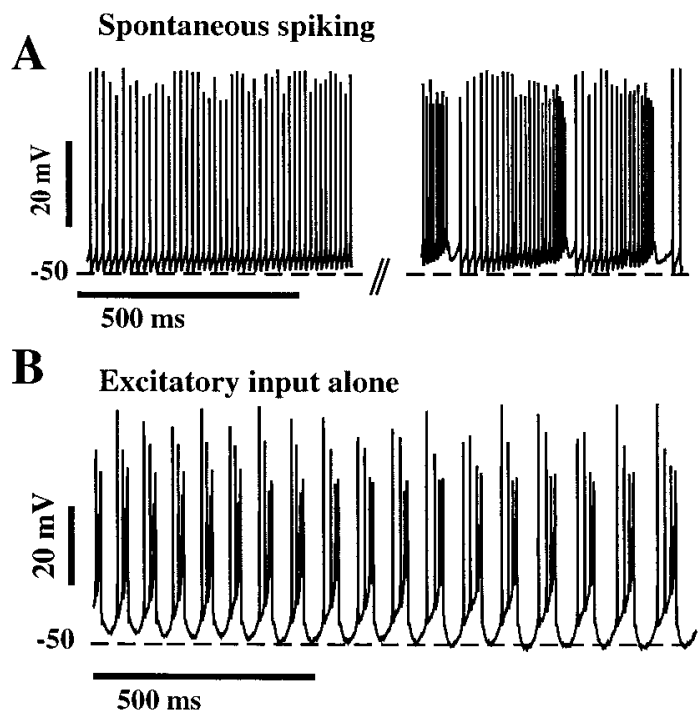

C Mixed excitatory and inhibitory input

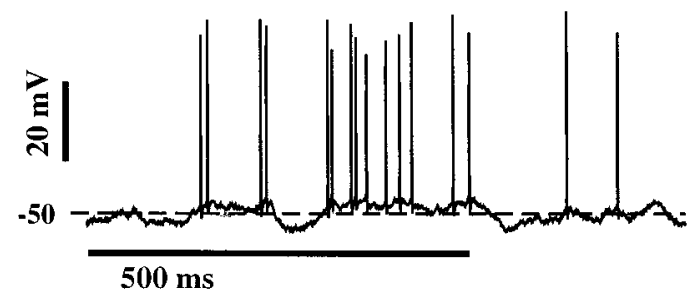

Figure 2. A, Spontaneous activity of a Purkinje cell during whole-cell recording in vitro while synaptic input was blocked pharmacologically is shown. Fast regular somatic spiking (left) is followed a few seconds later by spike bursting that is caused by dendritic calcium spikes (right). After a period of spike bursting, this cell became quiescent for a few seconds before the fast sodium spiking resumed (data not shown). $B$, When purely excitatory input was given with the dynamic clamp, intrinsic bursting activity sped up substantially. Spike timing was not related to the input under these conditions. $C$, The application of mixed inhibitory and excitatory conductances resulted in an ongoing irregular spike pattern characteristic of the in vivo state.

\section{Interspike interval lengths are related to the strength of inhibitory conductances}

Our modeling study (Jaeger et al., 1997) predicted that inhibitory synaptic conductances have a direct influence on the expected duration of intervals between somatic action potentials. Consistent with this prediction, the vertical dashed lines in Figure 4 indicate that periods of increased inhibition resulted in a cessation of spiking in the experimental data. This relationship is further quantified in Figure 5 in which we use the technique of spike-triggered averaging to compare the average levels of synaptic conductances associated with short, medium, and long interspike intervals. Figure 5, $A$ and $B$, reproduces the results of the same analysis reported previously for model data (Jaeger et al., 1997). Figure 5, $C$ and $D$, shows the experimental data. In both cases inhibitory synaptic conductances were larger, and $V_{m}$ was more hyperpolarized very early in the time course for long intervals compared with that for short intervals. This relationship was present in all cells analyzed $(n=8)$. Thus, as in the model, the length of the interspike intervals was correlated with the level of inhibitory input. A direct correlation of natural inhibitory input with prolonged interspike intervals was recently demonstrated in an elegant dual-recording study from interneurons and Purkinje cells (Häusser and Clark, 1997).

\section{Excitatory inputs have relatively little direct effect on spike initiation}

The spike-triggered averages shown in Figure 5 also allowed us to examine the relationship between somatic spiking and simulated excitatory synaptic conductances. In the model (Fig. $5 B$ ) there was virtually no change in the average level of excitatory conductance before, during, or after an action potential. This modeling result led to the somewhat surprising suggestion that spike initiation does not reflect a previous increase in excitatory input (Jaeger et al., 1997). Furthermore, the mean level of excitation was not related to the control of ISI duration. The spike-triggered averages obtained from physiological records in these experiments support the same conclusion (Fig. 5D). The level of excitatory conductances during an ISI was at no time correlated with ISI duration in any of eight cells analyzed.

The fact that excitatory inputs in the conductances we use are more frequent, smaller in amplitude, and shorter in duration than are inhibitory inputs may account for their ineffectiveness in controlling spike timing. Because of these statistical properties, the total excitatory conductance exhibits much less fluctuation than the total inhibitory conductance does (Fig. 4A). The chosen input properties are not arbitrary, because they were based on well established anatomical and physiological data (see Materials and Methods). Nevertheless, our assumption of independent stochastic activation of synapses represents only one possible input condition. Many excitatory inputs firing together at the same time does result in a precisely timed spike (De Schutter and Bower, 1994c). Whether and under what conditions such highly correlated inputs may occur cannot be answered by the present study.

\section{The level of excitatory synaptic conductance strongly affects the rate of spiking via the activation of intrinsic currents}

The absence of a correlation between excitatory inputs and the duration of individual interspike intervals does not imply that excitatory input is without function. In fact, the model predicts that Purkinje cells should show a dramatic increase in spike rate for only moderate increases in the amount of excitation received (De Schutter and Bower, 1994b). Such a high gain in the change of output spike rate would make the Purkinje cell a very sensitive rate-coding device for the average level of excitatory input received (Jaeger et al., 1997). In the model, this high gain in the spike output rate is primarily caused by dendritic P-type $\mathrm{Ca}$ channel activation (Jaeger et al., 1997). To investigate the gain function of the output spike rate in vitro, we increased the amount of baseline excitation in the dynamic current clamp (Fig. 6). This was done by adding a constant excitatory conductance such that the fluctuations in the synaptic conductances remained identical. An increase of 10 or $30 \%$ in the mean excitatory conductance led to an increase in spike rate of 43 or $115 \%$, respectively (average of eight cells). Thus, as predicted by the model, the in vitro recordings showed a high gain function for the rate coding of excitatory input levels. The increases in spike rate of 43 and $115 \%$ were accompanied by a depolarizing shift in the average $V_{m}$ of 1.3 and $3.8 \mathrm{mV}$, respectively.

The depolarization and increase in spiking seen with increased excitatory conductance were not caused by an inward current via the simulated synaptic conductance. In fact the mean synaptic current remained outward with nearly identical amplitude for the lowest and highest amount of excitation $(-0.45$ and $-0.41 \mathrm{nA}$, respectively; $n=8$ cells). This result was also predicted by the model. The lack of an increased inward synaptic current with an 

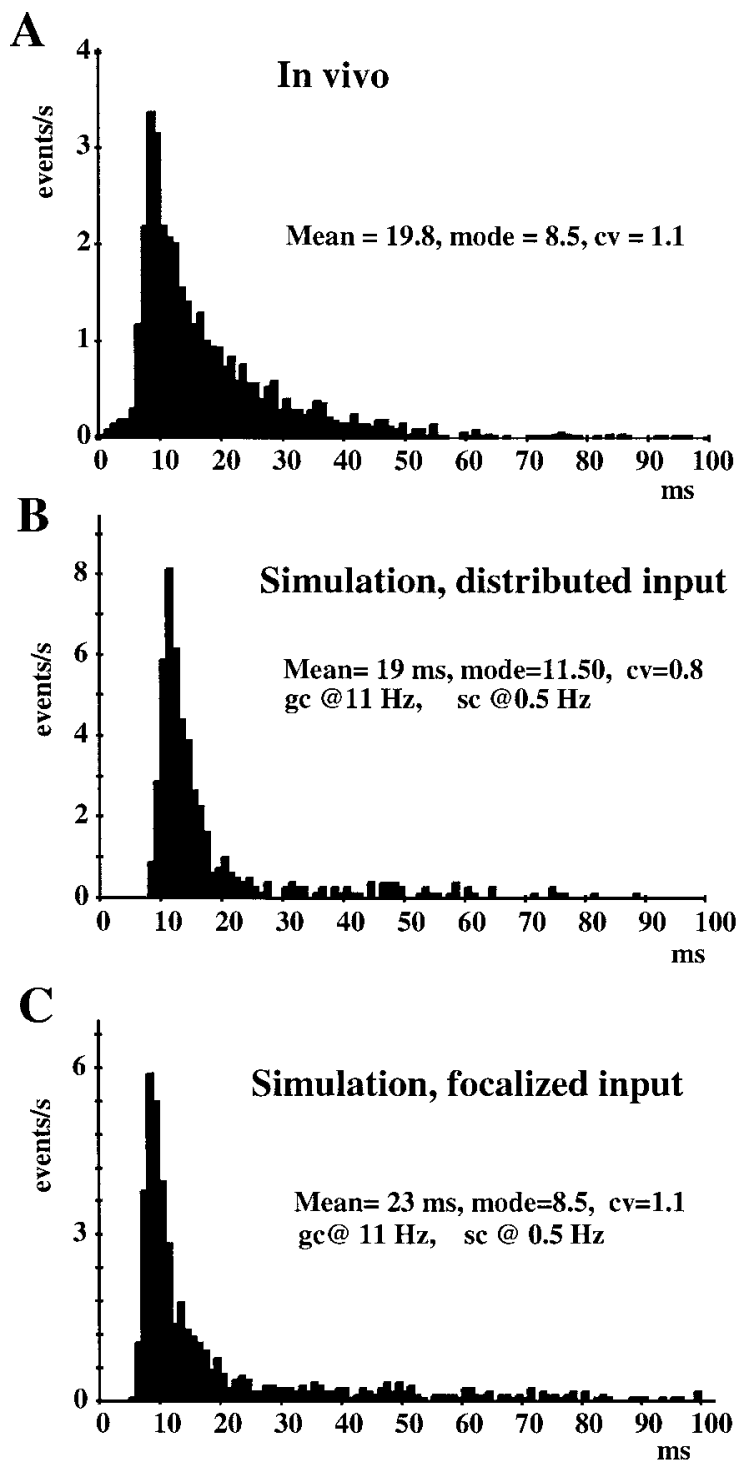

D

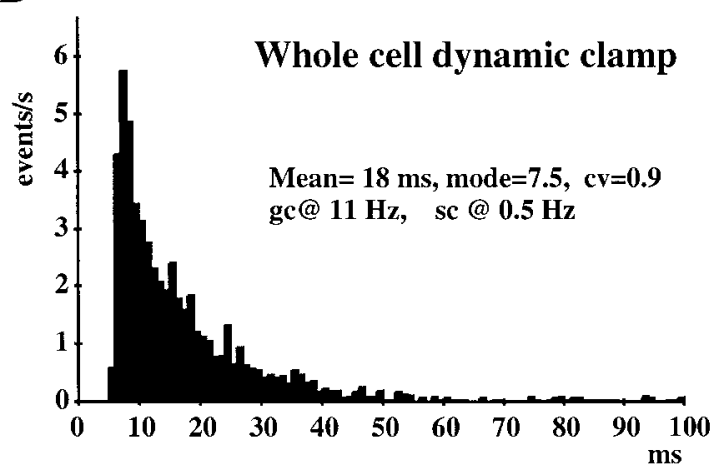

Figure 3. ISI histograms of Purkinje cell spiking for an in vivo recording $(A)$, in the model with distributed $(B)$ and focalized $(C)$ input, and during dynamic current clamping in vitro $(D)$. The ISI distributions were overall similar in all conditions, showing a strong modal peak at $\sim 10 \mathrm{msec}$ and a tail of long intervals. The total input conductances in the model and under the dynamic current-clamp condition were identical. They were obtained from a computer simulation in which excitatory granule cell $(g c)$ synapses were activated randomly with a mean rate of $11 \mathrm{~Hz}$ and inhibitory stellate cells $(s c)$ were activated randomly with a mean rate of 0.5 $\mathrm{Hz}$. The in vivo data were obtained with an extracellular recording from crus IIA in the anesthetized rat [methods described in Jaeger and Bower
A

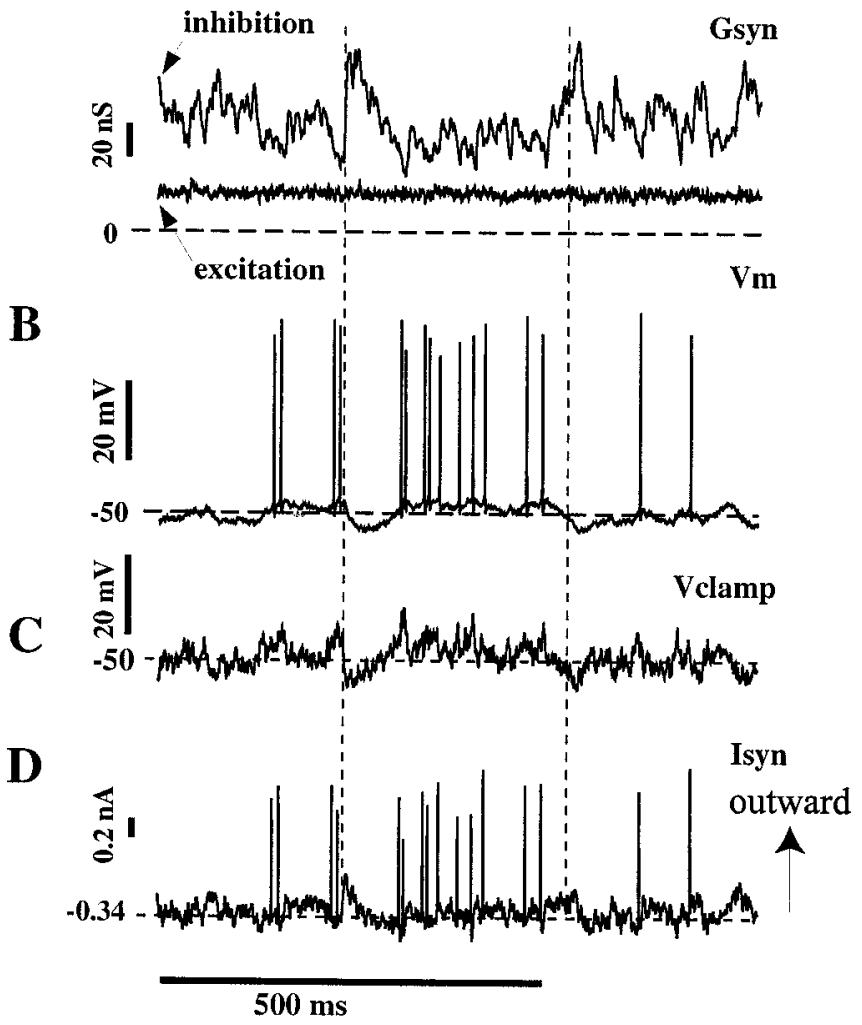

Figure 4. Response of an in vitro neuron to the application of modelbased synaptic conductances. $A$, The applied inhibitory and excitatory synaptic conductance $\left(G_{\mathrm{syn}}\right)$ is shown. $B, C$, The somatic membrane potential $\left(\mathrm{V}_{\mathrm{m}}\right)$ follows the fluctuations in the clamping potential $\left(V_{\text {clamp }}\right)$ given by the input conductances. Spiking is inhibited during periods of increased inhibition (vertical dashed lines). $D$, The injected current $\left(I_{\text {syn }}\right)$ had an average gain of $0.25 \mathrm{nA}$ for each millivolt of deviation between $V_{\text {clamp }}$ and $\mathrm{V}_{\mathrm{m}}$. This gain was time-varying (proportional to the sum of excitatory and inhibitory conductances). The mean amplitude of injected current was $-0.34 \mathrm{nA}$, hyperpolarizing the cell.

increase in excitatory conductance proves that intrinsic inward current is responsible for the additional depolarization. As the cell is allowed to depolarize more by a depolarizing shift in the combined synaptic reversal potential, inward voltage-dependent currents are activated more strongly. The P-type calcium channel in particular shows a steep activation increase in the relevant voltage range of -50 to $-35 \mathrm{mV}$ (Regan, 1991). This increased intrinsic inward current again pushes the cell beyond the synaptic reversal potential, and a net outward synaptic current is maintained. In principle, this mechanism allows neurons to achieve arbitrarily high gain functions in spike rate for small increases in excitatory input. Decreases in inhibitory input were similarly effective in increasing spike rates (data not shown). This is not surprising, because decreases in inhibition have a similar effect on shifting the combined synaptic reversal potential as do increases in excitation. The appearance of dendritic calcium spikes in the absence of inhibitory input (Fig. $2 A, B$ ) indicates that outward

(1994)]. The small number of very short ISIs in the in vivo distribution is attributable to complex spikes resulting from climbing fiber inputs and was determined by separate complex spike discrimination (data not shown). This input pathway was not included in the modeling or dynamicclamp studies. 


\section{Spike triggered averaged: Simulation}

A

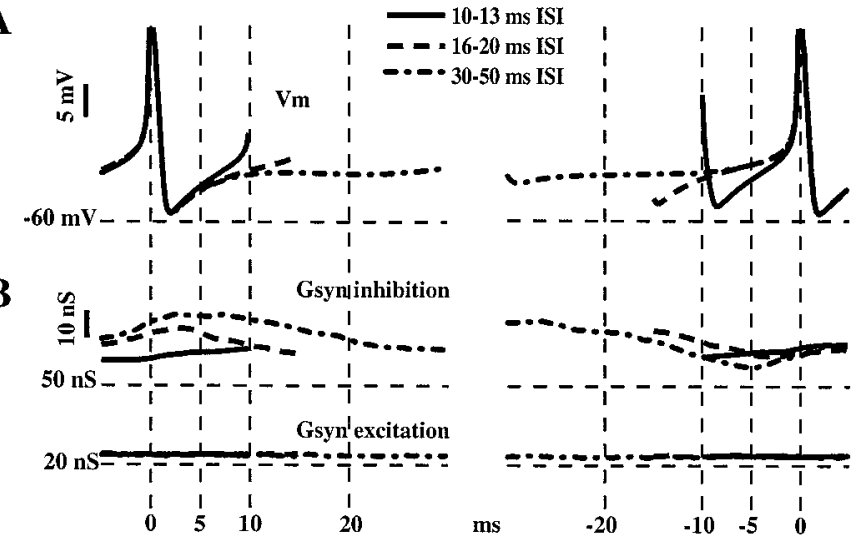

Spike triggered averages: Dynamic Current Clamp

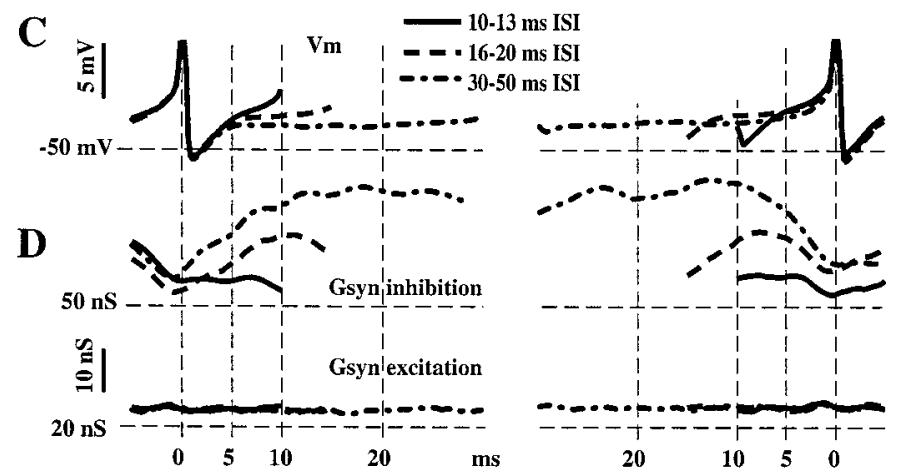

Figure 5. Spike-triggered averages of membrane potential $(A, C)$ and synaptic conductances $(B, D)$ in the model $(A, B)$ and with the dynamic clamp in vitro $(C, D)$. Spikes before or after ISIs with durations of $10-13$, $16-20$, or $30-50 \mathrm{msec}$ were separated into three groups to construct spike-triggered averages of membrane potential $\left(\mathrm{V}_{\mathrm{m}}\right)$, inhibitory synaptic conductance $\left(G_{\text {syn }}\right.$ inhibition), and excitatory synaptic conductance $\left(G_{\text {syn }}\right.$ excitation). The data were obtained from $2.5 \mathrm{sec}$ of cell activity for the model and the dynamic clamp.

synaptic current is important in preventing a runaway activation of intrinsic inward current. The Purkinje cell model further suggests that intrinsic inward current is also balanced by intrinsic voltage and $\mathrm{Ca}$-gated $\mathrm{K}$ conductances.

\section{The precision of spike timing is dependent on the absolute amplitude of synaptic conductances}

The strong participation of intrinsic currents in mediating the effect of synaptic input poses the question of how precisely spike timing can still be controlled by the synaptic conductance pattern. The precision of spike timing has recently received much attention as a possible mechanism for neural coding (Mainen and Sejnowski, 1995; König et al., 1996). To test for the control of precise spike timing by synaptic conductances in Purkinje cells, we applied synaptic conductances of different amplitudes. Specifically, we applied synaptic input conductances with different absolute amplitudes while maintaining an identical time course in the trajectory of the combined reversal potential. This was achieved by multiplying both inhibitory and excitatory conductance patterns by constant gain factors of $0.5,1.0$, or 2.0.

By applying the same conductance traces repeatedly via the dynamic clamp, we could assess the relationship between a given simulated synaptic input and the precision of spike timing (Fig. 7). Spike rasters show that an increase in the synaptic gain results in an improved alignment of individual spikes between stimulus repetitions (Fig. 7A). This effect was quantified by a crosscorrelation analysis, in which the timing of spikes for successive pairs of stimulus repetitions was correlated (Fig. 7B). For all stimulus gains, we find a very sharp peak in the crosscorrelograms, indicating the presence of precisely timed spikes. The proportion of spikes precisely timed within $\pm 1 \mathrm{msec}$ was much higher for the input gain of $2(78 \%)$ than for the input gain of $0.5(50 \%$; average of four cells). In addition, several cells showed a total loss of correlation with the synaptic input at a gain of 0.5 by entering an oscillatory bursting pattern (data not shown). Thus the gain of 0.5 was near the minimum of synaptic conductance needed to prevent runaway activation of intrinsic currents. These findings indicate that at a sufficient amplitude of synaptic conductances, a substantial proportion of spikes can be timed precisely in relation to the input even in the presence of large intrinsic currents.

A striking effect of applying conductances with different gain factors was consistently found; increasing the conductance amplitude led to a decrease in spike rate (Fig. 7A). This effect further substantiates the presence of inward intrinsic currents that push the membrane potential above the synaptic reversal potential. The ability of this current to depolarize the cell is reduced in the presence of large synaptic conductances, because the amount of synaptic current that opposes the intrinsic current to push the cell away from the synaptic reversal potential is increased (Fig. $7 C$ ). In our data, the average $\mathrm{V}_{\mathrm{m}}$ for the input gain of 1 was $-47.7 \mathrm{mV}$, whereas for a gain of 2 , it was $-49.6 \mathrm{mV}(n=4$ cells). The resulting spike rates were 32 and $26 \mathrm{~Hz}$, respectively.

\section{Spiking rates are controlled independently of spiking precision}

The previous sections indicate that Purkinje cells are a sensitive rate encoder with respect to the balance of inhibition and excitation but that they can also initiate precisely timed spikes with respect to synaptic conductance fluctuations. We now ask the further question of whether these two aspects of coding information about the input are independent of each other. This question can be addressed by a more detailed analysis of the data with increasing amounts of excitatory input conductance (Fig. 6). This figure allows the important observation that the precise timing of particular spikes, which are present at a low level of excitation, remains largely intact when additional excitatory conductance is added (Fig. 6A). Because the synaptic reversal potential is more depolarized with increasing excitation, more spikes are added. The proportion of these additional spikes that are timed precisely within $\pm 1 \mathrm{msec}$ is identical to that seen at a low level of excitation. The cross-correlograms show that the proportion of spikes that occurred with a precision of $\pm 1 \mathrm{msec}$ was $66-67 \%$ for all amounts of excitation (average of eight cells) and was thus maintained at low and at high spike rates (Fig. 6B).

Taken together with the findings shown in Figure 7, these data suggest that spike rate and spike precision are primarily controlled by two different properties of the input. Spike rate is mainly a function of the relative level of excitation and inhibition, which determines the average combined reversal potential. In contrast, spike precision depends on the total amplitude of synaptic conductance changes. Therefore, Purkinje cells in principle can transfer information about the input via precisely timed spikes independent of spike rate. Whether information is actually 
A

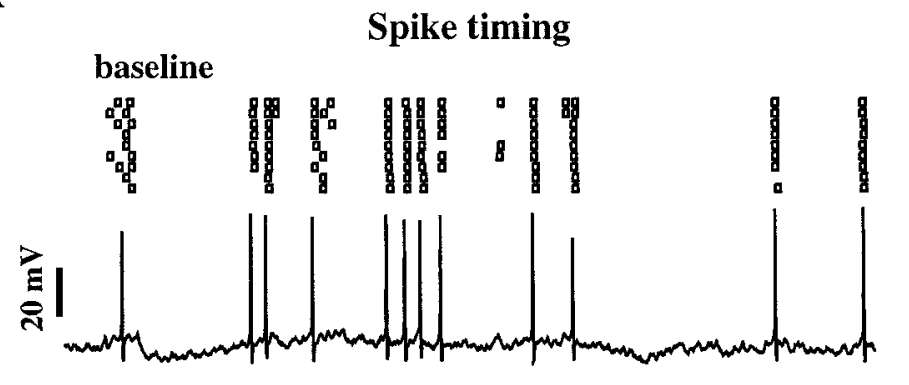

$+10 \%$ excitation
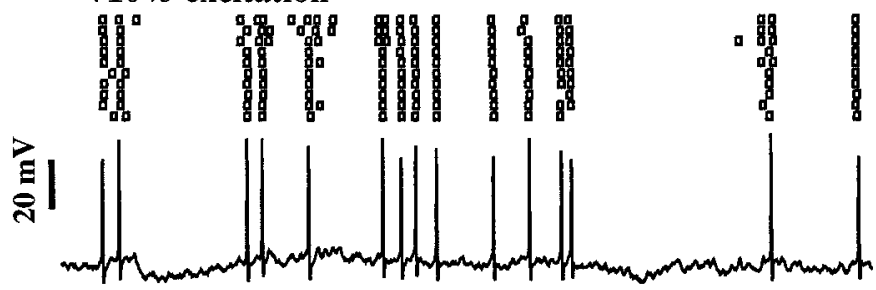

$+\mathbf{3 0} \%$ excitation

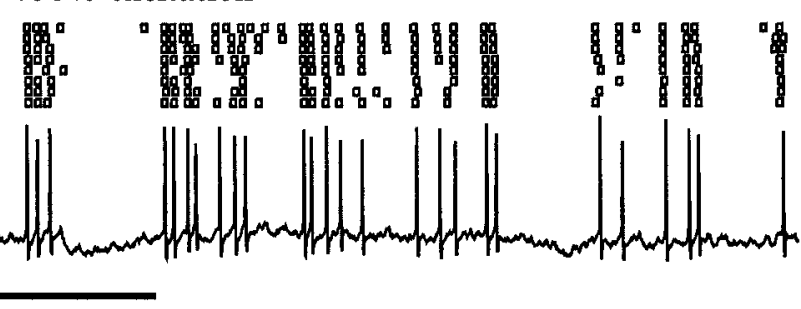

B

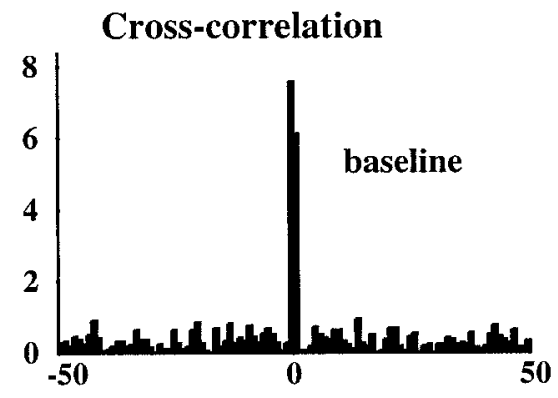

$100 \mathrm{~ms}$
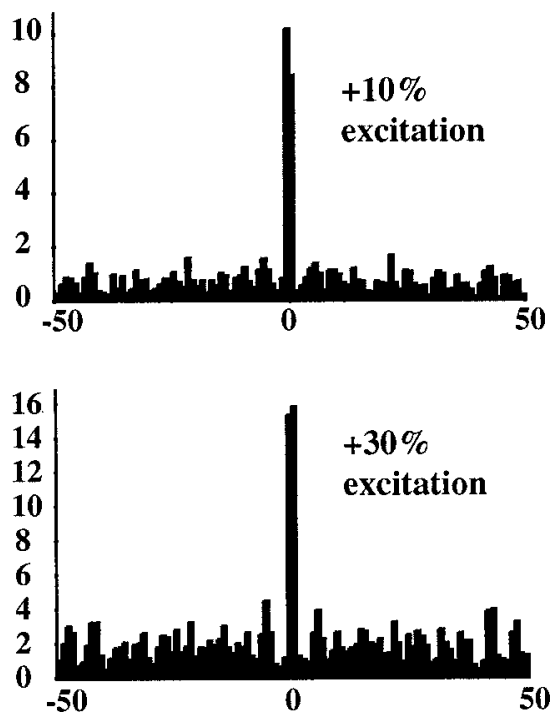

Figure 6. Spiking response of a single cell to increases in the constant component of simulated excitation. $A$, Dot rasters and single traces for three runs in which the amplitude of excitatory synaptic inputs was increased. Dot rasters indicate spike timing for repeated presentations of the same stimulus. The single $\mathrm{V}_{\mathrm{m}}$ trace below each dot raster illustrates the membrane potential trajectory for a typical response. $B$, Cross-correlations for each level of excitation. The cross-correlation was computed between subsequent responses to the same stimulus. The total conductance amplitude corresponded to a gain of 2.0 (see Fig. 7).

transferred via precisely timed spikes depends on the decoding mechanism at postsynaptic cells and the presence of precisely timed spikes across different Purkinje cells. These issues remain to be addressed in future studies.

\section{Variations in responses to applied inputs between Purkinje cells}

Finally, we used the dynamic current clamp to examine variations in the response of different in vitro Purkinje cells to the same simulated synaptic input. Figure 8 indicates that although each cell again responded consistently with a near-identical spike pattern for repeated presentations of the same stimulus, there were clear response differences between different cells. These differences included overall lower or higher spike rates in the response as well as different patterns of spiking. In particular, the amount of burstiness during periods of spiking was quite variable such that cells with overall low spike rates could show extremely rapid spiking at some times (Fig. $8 C$ ). Similar differences in spike patterns were observed in a previous modeling study (De Schutter and Bower, 1994a), when either the density of specific ion channels or the relative somatic or dendritic size of the cell was changed. These findings indicate that intrinsic conductances make an important contribution to spike timing. Because intrinsic currents are activated in a deterministic pattern by a repeated identical stimulus, the precision of spike timing in relation to the stimulus can be maintained. Because the dynamics of multiple interacting intrinsic currents is complex, the spike timing resulting from applied synaptic conductances may be a nontrivial function of fluctuations in the synaptic conductance. The diversity of observed spike responses teaches the important lesson that Purkinje cells may not be a homogeneous population of cells responding to inputs in an identical manner. Whether the inputoutput function of individual cells is the result of an adaptive learning process beyond the mere adjustment of synaptic strength remains to be studied. Recent experimental and modeling work in other cell types has clearly indicated that intrinsic currents can be dynamically regulated (Turrigiano et al., 1994, 1995; Liu et al., 1998).

\section{DISCUSSION}

\section{Testing the validity of modeling predictions}

In any modeling study, the usefulness and accuracy of simulation results are to a large degree dependent on the choice of modeling parameters (Bhalla and Bower, 1993). Because these parameters are never fully constrained by experimental data, the results of computer simulations aimed at replicating a biological system need to be carefully tested for their validity. On the other hand, modeling can clearly enhance our grasp of interacting dynamic variables, and only in a model can all state variables be accessed 
A

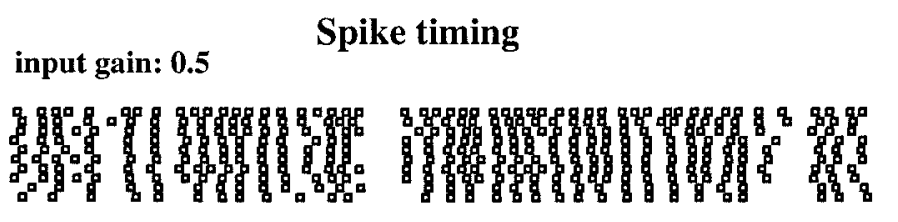

input gain: 1.0
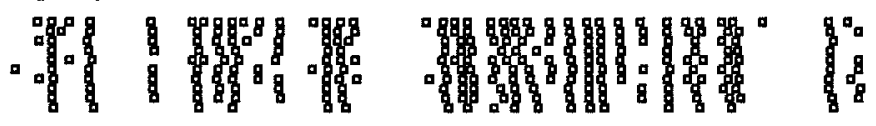

input gain: 2.0
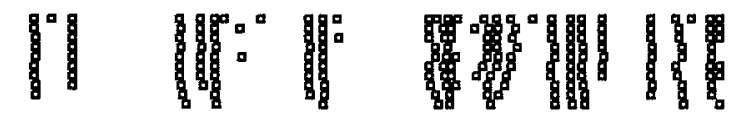

$100 \mathrm{~ms}$

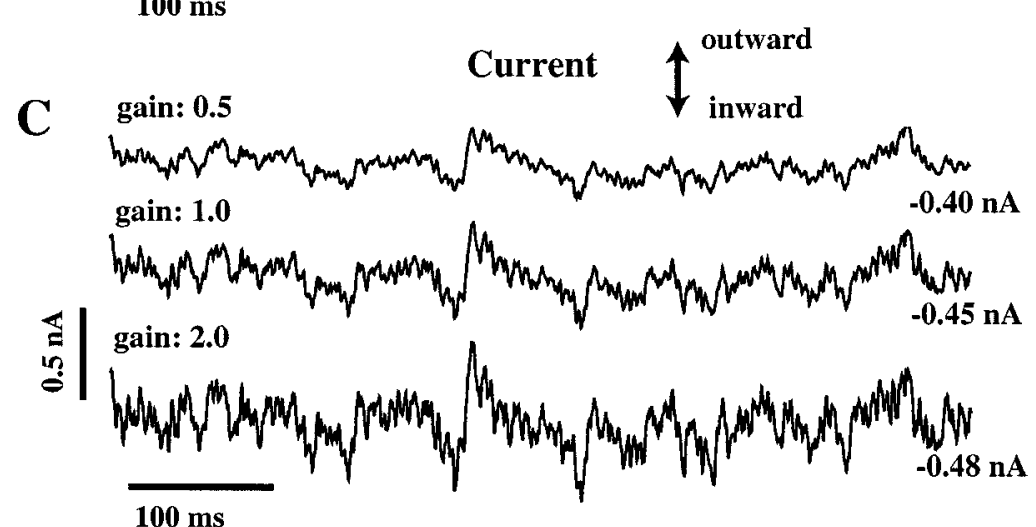

B
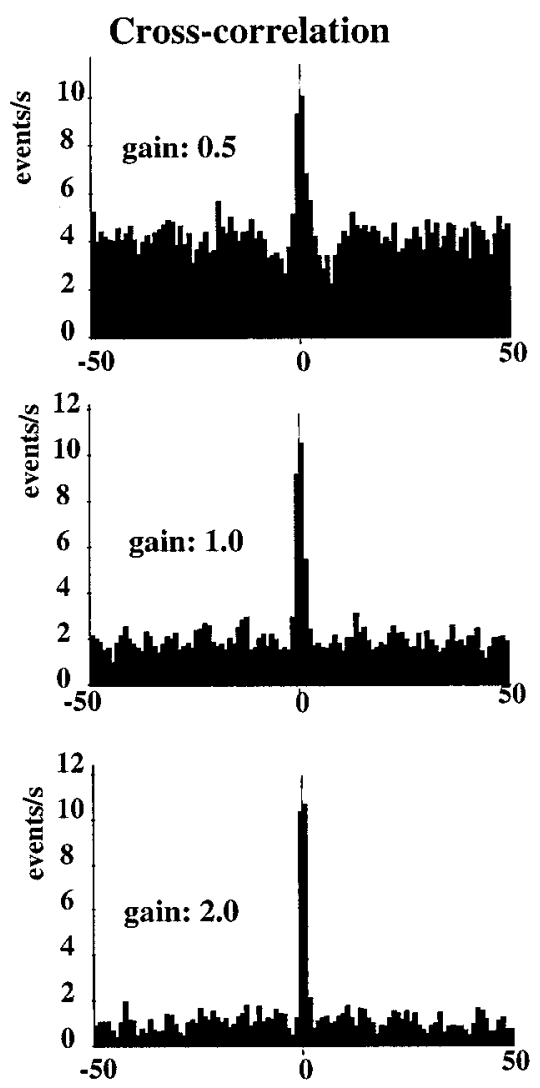

Figure 7. Spiking responses of a single cell with increasing input amplitudes. $A$, Dot rasters of spiking patterns with repeated presentations of the same input conductances multiplied by the indicated gain factors are shown. The spike frequency increased from 43 to $64 \mathrm{~Hz}$ when the input conductance was halved and decreased to $32 \mathrm{~Hz}$ when the input conductance was doubled. $B$, Cross-correlation histograms were computed by correlating spike times in each response with spike times of the next response. $C$, The fluctuations in the injected current increased proportionally with increasing input conductances; however, the mean currents changed only slightly from $-0.4 \mathrm{nA}$ for the gain of 0.5 to $-0.48 \mathrm{nA}$ for the gain of 2.0 . Inward current is plotted downward.

at the same time. The present study was designed to test modeling predictions about the integration of synaptic inputs in the presence of strong intrinsic currents, which we published previously (Jaeger et al., 1997). The best experimental method we could find to test our model predictions was to apply the synaptic conductances produced by the model to Purkinje cells in vitro by way of dynamic current clamping. In addition to testing our model predictions, this technique allowed us to pursue the question of how precisely spike timing can be controlled via synaptic input in a cell with strong intrinsic conductances. This issue of precisely timed spikes as a channel of neural coding has recently attracted much attention (Softky and Koch, 1993; Mainen and Sejnowski, 1995; König et al., 1996; Yuste and Tank, 1996; Shadlen and Newsome, 1998). Our finding that the control of spiking may to a large degree depend on intrinsic cell properties indicates that the spike coding of input may be quite different for neurons with different active properties. The experimental methods introduced here provide a means by which both the input and the output of a cell can be assessed at the same time and may thus prove useful in studying the control of spiking in various cell types. For Purkinje cells, our findings indicate that both rate coding and precise spike time coding can coexist at the same time. The specific interaction of synaptic conductances and intrinsic currents has important implications for cerebellar cortical function, as discussed below.

\section{Mechanisms underlying the synaptic control of Purkinje cell spiking}

\section{The influence of intrinsic conductances}

The experimental results reported here support our modeling prediction that Purkinje cells generate an intrinsic inward plateau current that pushes the cell above the combined synaptic reversal potential of excitation and inhibition. This intrinsic inward current then forces the net synaptic current to be outward. As in the model, we found that this relationship holds for the whole range of realistic spike rates (Fig. 6). The intrinsic inward current is unstable and results in spontaneous bursting in the absence of synaptic input (Fig. 2). Thus an ongoing balance of inhibitory and excitatory input is required to result in an irregular spike train typically seen in vivo. Our experiments cannot identify the types of ion channels responsible for the plateau depolarization in vitro. Previous studies show that the major candidates for a persistent inward current are given by a dendritic P-type $\mathrm{Ca}$ conductance and a somatic persistent Na conductance (Llinás and Sugimori, 1992). The model indicates that a large contribution of P-type Ca current is inevitable on the basis of the voltage dependence of this channel (Regan, 1991). In fact, in the model this current needs to be counterbalanced by Ca-dependent K currents [Jaeger et al. (1997), their Fig. 6] as well as by synaptic current. In the 


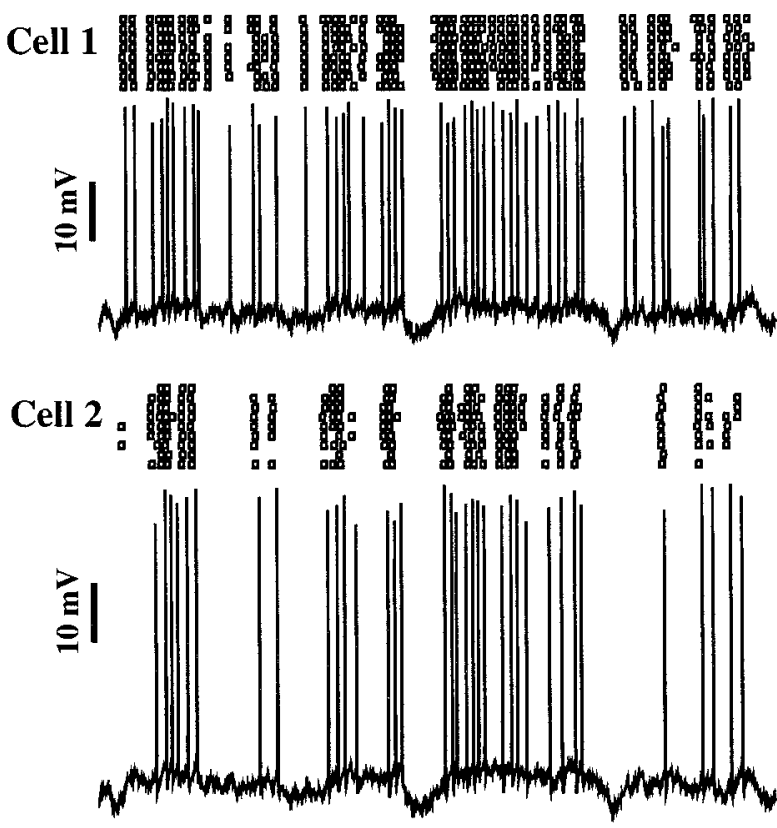

Cell 3

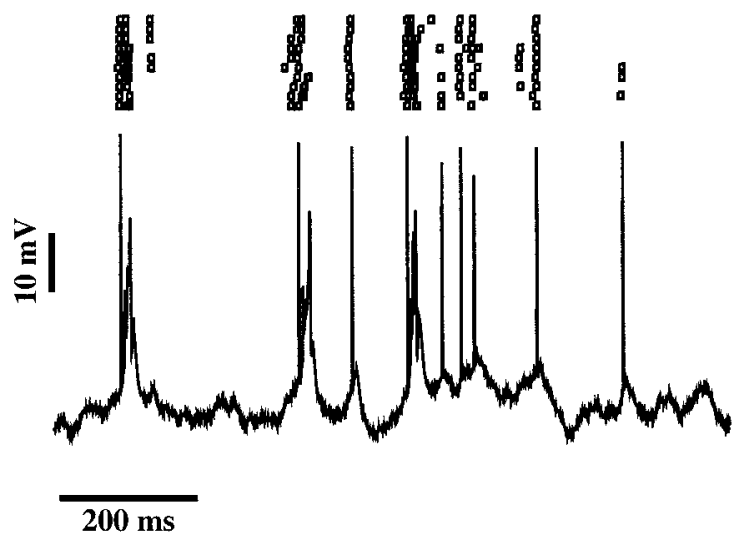

Figure 8. Comparison of spiking behavior for three different recorded Purkinje cells. All cells were stimulated with the identical input conductances. It can be seen that each cell has a very similar response for repeated presentation of the stimulus, whereas the response of different cells to the same stimulus can be quite different.

model, the interplay of dendritic $\mathrm{Ca}$ and $\mathrm{K}$ conductance activation leads to a complex time course of synaptically induced voltage transients. The role of intrinsic dendritic currents therefore goes much beyond a constant plateau depolarization. The detailed model predictions in this regard could not be tested in the present study because we cannot measure the time course of dendritic conductances. The variability in spike patterns between different cells recorded in vitro with the same input conductances (Fig. 8) suggests that the interaction of intrinsic and synaptic currents follows a different time course in different cells.

Persistent somatic sodium currents also provide a considerable inward current in the model and possibly in vitro. The model indicates, however, that these sodium currents are deactivated during spike afterhyperpolarization, and a depolarized dendrite is required to initiate the next spike (Jaeger et al., 1997).

\section{The role of inhibition}

Our experimental results show that a tonic baseline of inhibitory inputs needs to be present to allow the spike mode observed in vivo (Jaeger and Bower, 1994). This tonic input is required as otherwise intrinsic depolarizing currents push Purkinje cells into a burst mode that is not usually present in vivo. Our data show that the mean combined reversal potential of inhibition and excitation has to fall into a narrow range below spike threshold to allow natural spike patterns. This range in reversal potential is obtained when there are on the order of 20 times as many unitary excitatory as there are inhibitory inputs over a given time period.

A second important prediction of the model was also borne out in the experimental data, namely, that the time-varying level of inhibitory conductance has a direct control over the length of interspike intervals (Fig. 5). This finding is supported by the recent demonstration of a strong effect of the input from single inhibitory interneurons on Purkinje cell spike intervals (Häusser and Clark, 1997). These authors find that even the activation of interneurons in the outer molecular layer can have such an effect, and thus spike time control is not limited to basket cell inputs onto the soma. Basket cell input was not included in our modeling study, and further studies are needed to assess its influence in detail.

\section{Spike rate coding and spike time coding}

We find that Purkinje cells are very sensitive rate encoders in that small changes in the balance of excitation and inhibition lead to large changes in spike rate (Fig. 6). The larger changes in output than in input rate represent an amplification function. This amplification is carried by the activation of intrinsic plateau currents. Independent of spike rate, precise spike timing is found to be mediated via rapid fluctuations in synaptic conductances when the overall amplitude of synaptic conductance is high (Fig. 7). This spike precision in our data is limited to a narrow window of maximally $\pm 3 \mathrm{msec}$, making it a distinctly different process from rate coding. Thus it seems possible that the overall spike rate and the timing of individual spikes may transmit different signals to the next level of neural processing. Unfortunately, we are still almost entirely ignorant about the decoding of Purkinje cell spike trains by the neurons in the deep cerebellar nuclei to which they project.

\section{Significance for cerebellar network function Reconsidering inhibition}

Our results indicate that constant inhibitory input is required in the control of Purkinje cell spiking and that inhibitory input makes an important contribution to spike timing as well as to spike rate control. In contrast, most recent efforts of modeling cerebellar cortical network function make no use of inhibitory inputs to Purkinje cells (Kawato and Gomi, 1992; Buonomano and Mauk, 1994; Schweighofer et al., 1996). This may be inspired by the view of neurons as summation devices, in which the presence of inhibitory input is equivalent to a reduced level of excitatory input. Our data indicate that Purkinje cells cannot operate in this manner. We conclude that pauses in Purkinje cell spiking are necessarily coupled to the presence of inhibitory input. (An exception would be a pause in spiking because of $\mathrm{K}$ conductance activation after dendritic calcium spiking.) Such pauses in spiking may be of great significance, because Purkinje cells are themselves inhibitory neurons. Thus, a pause in spiking would disinhibit neurons in the deep cerebellar nuclei and lead to a positive output from the cerebellum. Pauses in spiking were found to be a dominant Purkinje cell response after somatosensory stimulation in anesthetized rats. This was true even along the 
center of an excited "beam" of parallel fibers (Bower and Woolston, 1983).

\section{The indirect effects of parallel fiber inputs}

Our experiments support the model prediction that parallel fiber (pf) inputs have a much more indirect effect on Purkinje cell behavior than is usually assumed. Since the first anatomical descriptions of the granule cell-parallel fiber system, most theories of cerebellar function (e.g., Marr, 1969; Fujita, 1982) have assumed that sets of activated pf synapses directly force Purkinje cells to cross spike threshold. Our model analysis, now supported by the experimental results presented here, suggests that pf inputs do not directly produce somatic action potentials. Instead, continuous pf input contributes one-half of a partial voltage-clamp mechanism, which stabilizes $\mathrm{V}_{\mathrm{m}}$ at the combined reversal potential of inhibition and excitation. The trajectory of the combined reversal potential and the total conductance amplitude in turn regulate the activation of large intrinsic voltage-dependent conductances. The activation of intrinsic inward currents then depolarizes the cell and leads to the initiation of somatic spikes.

\section{What do the parallel fibers do?}

Viewing parallel fiber synapses as modulating the state of dendritic conductances suggests a major change in how to think about cerebellar cortex (Bower, 1997a). The data presented here suggest that parallel fiber input acts in the context of counterbalancing inhibition. In this way parallel fibers can be seen as playing a more modulatory role, as we originally proposed (Bower and Woolston, 1983). We do, however, believe that the granule cell synapses associated with the ascending granule cell axon can produce a direct excitatory drive on Purkinje cells (Bower and Woolston, 1983; Jaeger and Bower, 1994; Gundappa-Sulur et al., 1999). Recent cerebellar circuit simulations suggest that in addition to providing multiple and synchronous excitatory synaptic inputs, synapses of the ascending axon may activate too quickly after mossy fiber inputs to be counterbalanced by inhibition via local interneurons (F. Santamaria and J. M. Bower, unpublished observations). An elegant experimental demonstration of the predominant activation of Purkinje cells via ascending granule cell axons was recently seen with voltage-sensitive dye imaging in a guinea pig whole-brain preparation (Cohen and Yarom, 1998). In these experiments, electrical stimulation of a patch of granule cells resulted in the activation of overlying Purkinje cells but not along the extended beam of activated parallel fibers.

\section{The challenge of understanding cerebellar cortical computation}

The specialized architecture of cerebellar cortex suggests that a particular computational algorithm may take place in this structure (Bloedel, 1992; Bower, 1997b). The strong interaction of synaptic input and intrinsic conductances that we found in our model is supported by the present experimental findings. Because this interaction changes the amplitude and time course of Purkinje cell responses to synaptic input, active properties likely play an important role in the implementation of the cerebellar computational algorithm. One key challenge in further elucidating cerebellar computation consists of determining the control of spiking in other cerebellar cell types and in the combined network. We expect that realistic network modeling in conjunction with experimental testing will be the method of choice in tracing the complex dynamics of cerebellar computation.

\section{REFERENCES}

Albus JS (1971) A theory of cerebellar function. Math Biosci 10:25-61. Barbour B (1993) Synaptic currents evoked in Purkinje cells by stimulating individual granule cells. Neuron 11:759-769.

Barry PH (1994) JPCalc, a software package for calculating liquid junction potential corrections in patch-clamp, intracellular, epithelial and bilayer measurements and for correcting junction potential measurements. J Neurosci Methods 51:107-116.

Barry PH, Lynch JW (1991) Liquid junction potentials and small cell effects in patch-clamp analysis. J Membr Biol 121:101-117.

Bhalla US, Bower JM (1993) Exploring parameter space in detailed single neuron models: simulations of the mitral and granule cells of the olfactory bulb. J Neurophysiol 69:1948-1965.

Bloedel JR (1992) Functional heterogeneity with structural homogeneity: how does the cerebellum operate? Behav Brain Sci 15:666-678.

Bower JM (1997a) Control of sensory data acquisition. Int Rev Neurobiol 41:489-513.

Bower JM (1997b) Is the cerebellum sensory for motor's sake, or motor for sensory's sake: the view from the whiskers of a rat? Prog Brain Res 114:483-516.

Bower JM, Woolston DC (1983) Congruence of spatial organization of tactile projections to granule cell and Purkinje cell layers of cerebellar hemispheres of the albino rat: vertical organization of cerebellar cortex. J Neurophysiol 49:745-766.

Buonomano DV, Mauk MD (1994) Neural network model of the cerebellum: temporal discrimination and the timing of motor responses. Neural Comput 6:38-55.

Cohen D, Yarom Y (1998) Patches of synchronized activity in the cerebellar cortex evoked by mossy-fiber stimulation: questioning the role of parallel fibers. Proc Natl Acad Sci USA 95:15032-15036.

De Schutter E, Bower JM (1994a) An active membrane model of the cerebellar Purkinje cell. I. Simulation of current clamp in slice. J Neurophysiol 71:375-400.

De Schutter E, Bower JM (1994b) An active membrane model of the cerebellar Purkinje cell. II. Simulation of synaptic responses. J Neurophysiol 71:401-419.

De Schutter E, Bower JM (1994c) Simulated responses of cerebellar Purkinje cells are independent of the dendritic location of granule cell synaptic inputs. Proc Natl Acad Sci USA 91:4736-4740.

Fujita M (1982) Adaptive filter model of the cerebellum. Biol Cybern 45:195-206.

Gundappa-Sulur G, De Schutter E, Bower JM (1999) Ascending granule cell axon: an important component of cerebellar cortical circuitry. J Comp Neurol 408:580-596.

Harvey RJ, Napper RMA (1988) Quantitative study of granule and Purkinje cells in the cerebellar cortex of the rat. J Comp Neurol 274:151-157.

Häusser M, Clark BA (1997) Tonic synaptic inhibition modulates neuronal output pattern and spatiotemporal synaptic integration. Neuron 19:665-678.

Jaeger D, Bower JM (1994) Prolonged responses in rat cerebellar Purkinje cells following activation of the granule cell layer: an intracellular in vitro and in vivo investigation. Exp Brain Res 100:200-214.

Jaeger D, De Schutter E, Bower JM (1997) The role of synaptic and voltage-gated currents in the control of Purkinje cell spiking: a modeling study. J Neurosci 17:91-106.

Kawato M, Gomi H (1992) A computational model of four regions of the cerebellum based on feedback-error learning. Biol Cybern 68:95-103.

König P, Engel AK, Singer W (1996) Integrator or coincidence detector? The role of the cortical neuron revisited. Trends Neurosci 19:130-141.

Liu Z, Golowassch J, Marder E, Abbott LF (1998) A model neuron with activity-dependent conductances regulated by multiple calcium sensors. J Neurosci 18:2309-2320.

Llinás R, Sugimori M (1980a) Electrophysiological properties of in vitro Purkinje cell somata in mammalian cerebellar slices. J Physiol (Lond) 305:171-195.

Llinás R, Sugimori M (1980b) Electrophysiological properties of in vitro Purkinje cell dendrites in mammalian cerebellar slices. J Physiol (Lond) 305:197-213.

Llinás R, Sugimori M (1992) The electrophysiology of the cerebellar Purkinje cell revisited. In: The cerebellum revisited (Llinás R, Sotelo C, eds), pp 167-181. New York: Springer.

Mainen ZF, Sejnowski TJ (1995) Reliability of spike timing in neocortical neurons. Science 268:1503-1506. 
Marr D (1969) A theory of cerebellar cortex. J Physiol (Lond) 202:437-471.

Palay SL, Chan-Palay V (1974) Cerebellar cortex: cytology and organization. Berlin: Springer.

Pusch M, Neher E (1988) Rates of diffusional exchange between small cells and a measuring patch pipette. Pflügers Arch 411:204-211.

Rall W (1959) Branching dendritic trees and motoneuron membrane resistivity. Exp Neurol 1:491-527.

Rapp M, Segev I, Yarom Y (1994) Physiology, morphology and detailed passive models of guinea-pig cerebellar Purkinje cells. J Physiol (Lond) 474:101-118.

Regan LJ (1991) Voltage-dependent calcium currents in Purkinje cells from rat cerebellar vermis. J Neurosci 11:2259-2269.

Robinson HPC, Kawai N (1993) Injection of digitally synthesized synaptic conductance transients to measure the integrative properties of neurons. J Neurosci Methods 49:157-165.

Schweighofer N, Arbib MA, Dominey PF (1996) A model of the cerebellum in adaptive control of saccadic gain. I. The model and its biological substrate. Biol Cybern 75:19-28.

Shadlen MN, Newsome WT (1998) The variable discharge of cortical neurons: implications for connectivity, computation, and information coding. J Neurosci 18:3870-3896.
Sharp AA, O’Neil MB, Abbott LF, Marder E (1993) The dynamic clamp: artificial conductances in biological neurons. Trends Neurosci 16:389-394.

Softky WR, Koch C (1993) The highly irregular firing of cortical cells is inconsistent with temporal integration of random EPSPs. J Neurosci 13:334-350.

Spruston N, Jaffe DB, Williams SH, Johnston D (1993) Voltage- and space-clamp errors associated with the measurement of electrotonically remote synaptic events. J Neurophysiol 70:781-802.

Sultan F, Bower JM (1998) Quantitative Golgi study of the rat cerebellar molecular layer interneurons using principal component analysis. J Comp Neurol 393:353-373.

Turrigiano G, Abbott LF, Marder E (1994) Activity-dependent changes in the intrinsic properties of cultured neurons. Science 264:974-977.

Turrigiano G, LeMasson G, Marder E (1995) Selective regulation of current densities underlies spontaneous changes in the activity of cultured neurons. J Neurosci 15:3640-3652.

Vincent P, Armstrong CM, Marty A (1992) Inhibitory synaptic currents in rat cerebellar Purkinje cells: modulation by postsynaptic depolarizations. J Physiol (Lond) 456:453-471.

Yuste R, Tank DW (1996) Dendritic integration in mammalian neurons, a century after Cajal. Neuron 16:701-716. 